\title{
Multiwavelength data for bright active galaxies
}

\author{
Areg M. Mickaelian ${ }^{1}$, Hayk V. Abrahamyan ${ }^{1}$, Gurgen M. Paronyan ${ }^{1}$ \\ and Gohar S. Harutyunyan ${ }^{2}$ \\ ${ }^{1}$ Byurakan Astrophysical Observatory (BAO), Byurakan 0213, Aragatzotn province, Armenia \\ email: aregmick@aras.am, abrahamyanhayk@gmail.com, pgurgen@bao.sci.am \\ ${ }^{2}$ Yerevan State University (YSU), A. Manoogian 1, Yerevan 0025, Armenia \\ email: goharutyunyan@gmail.com
}

\begin{abstract}
The spectral energy distribution (SED) gives a complete picture of the radiation of space objects and may result in correct classifications compared to those based only on optical (or other local) spectra. This is especially crucial for active galaxies, both AGN and Starbursts (SB). For this, multiwavelength (MW) data are needed taken from available surveys and catalogs. We have cross-correlated the Catalogue of quasars and active galaxies with all-sky or large-area MW catalogues, such as X-ray ROSAT (BSC and FSC), UV GALEX (MIS and AIS), optical APM, MAPS, USNO-B1.0, GSC 2.3.2, and SDSS DR8, NIR 2MASS, MIR/FIR WISE, IRAS (PSC and FSC) and AKARI (IRC and FIS), radio GB6, NVSS, FIRST, and WENSS. We have established accurate positions and photometry for a few thousands of objects that appeared in the catalog with poor data, as well as achieved the best astrometric and photometric data for all objects. This allowed correct cross-correlations and establishing correct MW data for these objects. As a result, we obtained 34 photometric points from X-rays to radio and using VO tools built SEDs for some 10,000 bright objects. Some data from other surveys were also used, such as Chandra, XMM, Spitzer, etc. All objects were grouped into several forms of SED and were compared to the known optical classes given in the catalog (QSO, BLL, Sy1, Sy1.2-1.9, Sy2, LINER, SB, and HII). This allowed reveal obscured AGN, as well as find previously misclassified objects. A homogeneous classification for these objects was established. The first part of this project is presented; establishment of accurate positions and photometry and cross-correlations with MW catalogs.
\end{abstract}

Keywords. catalogs, surveys, galaxies: active, galaxies: starburst

\section{The Catalogue of active galaxies and its statistical use}

The Catalogue of quasars and active nuclei (13th edition; Veron-Cetty \& Veron 2010; hereafter VCV-13) is the fullest collection of AGN (and other active galaxies). It contains 168,941 objects, including 133,336 QSO, 1,374 BL Lac objects, and 34,231 active galaxies (including 16,517 Sy1). Most of objects come from SDSS DR7 (Abazajian et al. 2009) and 2QZ/6QZ (Croom et al. 2004) surveys. The latest SDSS-based (SDSS-I and SDSS-II) QSO catalog was published by Schneider et al. (2010) and contains 105,783 spectroscopically confirmed QSOs over $9380 \mathrm{deg}^{2}$ surface. A Large quasar astrometric catalogue (LQAC) was recently built by Souchay et al. (2009) based on 12 optical and radio QSO catalogues. It contains 113,666 objects. In addition, Massaro et al. (2009) have published a multi-frequency catalogue of blazars with the fullest collection (3061 objects) of BL Lac and flat radio spectrum QSOs (last update: 1 July 2011). All these lists partially cover each other and for the users it is not easy to have a full set of data or even carry out cross-correlations between these catalogs (mainly, due to positional uncertainties). 
Table 1. All-sky / large area catalogues and their data.

\begin{tabular}{|c|c|c|c|c|c|c|}
\hline Catalogs & | Range & Area, surface & $\mid$ Accuracy $\mid$ & Sources & | Release & | Reference \\
\hline ROSAT BSC & X-ray & All-sky & 60 as & 18806 & |1999-11-05 & Voges et al. 1999 \\
\hline ROSAT FSC & X-ray & l-sky & 60 as & 105924 & $2000-05-26$ & Voges et al. 2000 \\
\hline GALEX MIS & UV & All-sky & 2 as & 12600000 & 2011-03-14 & Bianchi et al. 2011 \\
\hline GALEX AIS & UV & All-sky & 2 as & 65300000 & 2011-03-14 & Bianchi et al. 2011 \\
\hline $\mathrm{APM}$ & opt & $\delta>-33^{\circ},|b|>20^{\circ}$ & $619 \mathrm{mas}$ & 166466987 & 2000-12-05 & McMahon et al. 2000 \\
\hline MAPS & opt & $\left|\delta>-33^{\circ},\right| b \mid>20^{\circ}$ & $519 \mathrm{mas}$ & 89234404 & 2003-07-00 & Cabanela et al. 2003 \\
\hline USNO-B 1.0 & opt & All-sky & $387 \mathrm{mas}$ & 1045913669 & 2005-11-17 & Monet et al. 2003 \\
\hline GSC 2.3 .2 & opt & All-sky & $258 \mathrm{mas}$ & 945592683 & 2007-04-19 & Lasker et al. 2008 \\
\hline $2 \mathrm{QZ} / 6 \mathrm{QZ}$ & opt & SGP / NGP, & $338 \mathrm{mas}$ & 49425 & 2006-06-01 & Croom et al. 2004 \\
\hline SDSS-II & opt & $\delta>0^{\circ},|b|>30^{\circ}$ & $67 \mathrm{mas}$ & 357175411 & 2009-06-03 & Abazajian et al. 2009 \\
\hline $2 \mathrm{MASS}$ & NIR & All-sky & $479 \mathrm{mas}$ & 470992970 & 2003-06-10 & Cutri et al. 2003 \\
\hline WISE & NIR/MIR & All-sky & $500 \mathrm{mas}$ & 257310278 & 2011-04-14 & Wright et al. 2010 \\
\hline IRAS PSC & MIR/FIR & All-sky & 28 as & 245889 & 1994-01-29 & IRAS 1986 \\
\hline IRAS FSC & MIR/FIR & All-sky & 13 as & 173044 & 1993-02-24 & Moshir et al. 1989 \\
\hline AKARI IRC & MIR & All-sky & $300 \mathrm{mas}$ & 870973 & 2010-04-21 & Ishihara et al. 2010 \\
\hline AKARI FIS & FIR & All-sky & $800 \mathrm{mas}$ & 427071 & 2010-04-21 & Yamamura et al. 2010 \\
\hline GB 6 & radio & $0^{\circ}<\delta<+75^{\circ}$ & 210 as & 75162 & 1997-01-07 & Gregory et al.1996 \\
\hline NVSS & radio & $\delta>-40^{\circ}$, & 45 as & 1773484 & 2002-09-27 & Condon et al. 1998 \\
\hline FIRST & radio & $\delta>0^{\circ},|b|>3$ & 5 as & 811117 & 2011-04-03 & Becker et al. 2003 \\
\hline WENSS & radio & $+28^{\circ}<\delta<+76^{\circ}$ & 54 as & 229420 & 2004-05-14 & de Bruyn et al. 1998 \\
\hline
\end{tabular}

VCV-13 contains quasars with measured redshift known prior to July 1, 2009 (including SDSS DR7). As in the preceding editions, it does not give any information about absorption lines or X-ray/UV/IR properties. But absolute magnitudes and, when available, the 6 and $21 \mathrm{~cm}$ flux densities are given. The authors warn that the catalogue should not be used for any statistical analysis as it is not complete in any sense. One of the main problems is the absence of homogeneous accurate positional and photometric data: they are coming from the original papers and have extremely different accuracy and (in case of the magnitudes) bands (B, V, R, and other, making the magnitudes not useful for statistics). SDSS and 2QZ/6QZ provide reliable photometry, and in addition, homogeneous optical photometry is given in APM/MAPS/USNO/GSC catalogs measured from POSS1/POSS2. Absence of reliable photometry leads to misclassification of objects into QSO/Sy1 (as it is connected with the $\mathrm{M}_{B}=-22.25$ limit of the absolute magnitude), as well as impossibility of studies of luminosity functions, luminosity evolution, etc. Moreover, MW data for active galaxies would provide possibility to compare there properties, build MW SEDs, make up better classifications, and better understand their physics and evolution. Thus there is a need for cross-correlations of the AGN Catalogue with optical and MW data.

\section{Cross-correlations of AGN catalogue with MW data}

In general, cross-correlations lead to numerous misidentifications and doubtful associations. The problem arises due to selection of the search radius and final acceptance of found objects. Typically, a standard search radius is being given (like in Vizier and elsewhere) based on typical positional errors of the given catalog. We have developed a new method of calculation of the search radius based on $3 \sigma$ rms of individual objects/sources from each catalogue (Knyazyan et al. 2011). Such errors differ up to 10-15 times, so the traditional method leads to finding many associations out of $3 \sigma$ or losing many associations for objects with larger errors. Moreover, we use accurate rms values for optical catalogs to achieve the best matches (Mickaelian \& Sinamyan 2010). Accurate coordinates and magnitudes were derived using our methods developed for APM/MAPS/USNO/GSC data (Mickaelian \& Sinamyan 2010, Mickaelian et al. 2011). 

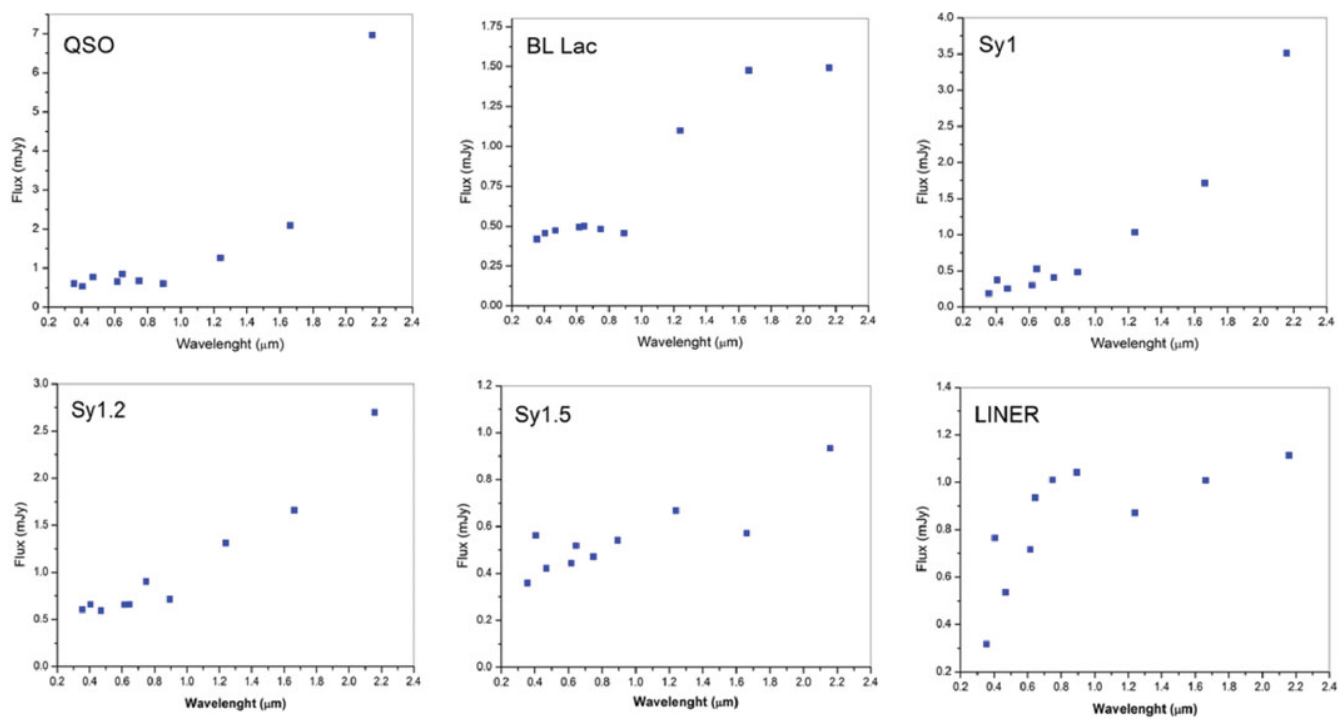

Figure 1. SEDs of active galaxies in the optical and NIR ranges by means of SDSS, POSS, and 2MASS photometric data.

Cross-correlations of the catalog of Quasars and AGN (VCV-13) have been carried out with the following surveys and catalogues given in Table 1.

\section{Results: MW data and SEDs for active galaxies}

MW SEDs have been built as shown in our previous papers (Sargsyan 2009; Hovhannisyan et al. 2011; Sargsyan et al. 2011). For the beginning, we have correlated POSS1/POSS2 B and R, SDSS ugriz and 2MASS JHK magnitudes. Different activity type objects have been selected: QSO, BLL, Sy1, Sy1.2, Sy1.5, and LINER. The results are shown in Figure 1. Slight differences are present in SEDs, however, average SEDs will reveal significant changes in different activity types.

\section{References}

Hovhannisyan A., Sargsyan, L. A., Mickaelian, A. M., \& Weedman, D. W. 2011, Ap, 54, 147

Knyazyan, A., Mickaelian, A., \& Astsatryan, H. 2011, Proc. CSIT-2011, in press

Massaro, E., Mickaelian, A. M., Nesci, R., \& Weedman, D. 2008, The Digitized First Byurakan Survey, ARACNE Editrice, Rome, Italy, $78 \mathrm{p}$.

Massaro, E., Giommi, P., Leto, C., et al. 2009, A\& $A$, 495, 691

Mickaelian, A. M., Nesci, R., Rossi, C., et al. 2007, A\&A, 464, 1177

Mickaelian, A. M. \& Sinamyan, P. K. 2010, MNRAS, 407, 681

Mickaelian, A. M., Mikayelyan, G. A., \& Sinamyan, P. K. 2011, MNRAS, 415, 1061

Sargsyan, L. A. 2009, Ap, 52, 377

Sargsyan, L., Weedman, D., Lebouteiller, V., et al. 2011, ApJ, 730, 19

Schneider, D. P., Richards, G. T., Hall, P. B., et al. 2010, AJ, 139, 2360

Souchay, J., Andrei, A. H., Barache, C., et al. 2009, A\&A, 494, 799

Veron-Cetty, M.-P. \& Veron, P. 2010, A\&SA, 518, 10 